\title{
Insights into the structural function of the complex of HIV-1 protease with TMC-126: molecular dynamics simulations and free-energy calculations
}

\author{
Dan Li • Ju-Guang Han • Hang Chen • Liang Li • \\ Run-Ning Zhao • Guang Liu • Yuhua Duan
}

Received: 18 December 2010 / Accepted: 26 July 2011 /Published online: 18 August 2011

(C) Springer-Verlag 2011

\begin{abstract}
The binding properties of the protein-inhibitor complex of human immunodeficiency virus type 1 (HIV-1) protease with the inhibitor TMC-126 are investigated by combining computational alanine scanning (CAS) mutagenesis with binding free-energy decomposition (BFED). The calculated results demonstrate that the flap region (residues 38-58) and the active site region (residues 23-32) in HIV-1 protease contribute $63.72 \%$ of the protease to the binding of the inhibitor. In particular, the mechanisms for the interactions of key residues of these species are fully explored and analyzed. Interestingly, the regression analyses show that both CAS and BFED based on the generalized Born model yield similar results, with a correlation coefficient of 0.94 . However, compared to CAS, BFED is faster and can decompose the per-residue binding free-energy contributions into backbone and sidechain contributions. The results obtained in this study are useful for studying the binding mechanism between receptor and ligand and for designing potent inhibitors that can combat diseases.
\end{abstract}

Keywords HIV-1 protease - TMC-126 · MM-PBSA/MMGBSA · Free-energy decomposition · Computational alanine scanning

D. Li $\cdot$ J.-G. Han $(\varangle) \cdot$ H. Chen $\cdot$ L. Li $\cdot$ R.-N. Zhao $\cdot$ G. Liu

National Synchrotron Radiation Laboratory,

University of Science and Technology of China,

Hefei 230029, People's Republic of China

e-mail: jghan@ustc.edu.cn

\section{Y. Duan}

National Energy Technology Laboratory,

United States Department of Energy,

Pittsburgh, PA 15236, USA

\author{
Abbreviations \\ PR Protease \\ PI Protease inhibitors \\ MD Molecular dynamics \\ PME Particle mesh Ewald \\ MM Molecular mechanics \\ GB Generalized Born \\ PB Poisson-Boltzmann \\ SA Surface area \\ rmsd Root-mean-square deviation
}

\section{Introduction}

Acquired immune deficiency syndrome (AIDS), which is induced by human immunodeficiency virus (HIV) infection, has become one of the major medical and humanitarian challenges. HIV-1 protease (PR), a member of the aspartyl protease family, is one of the most important enzymes targeted in research aimed at discovering new drugs to counter AIDS. It cleaves the nonfunctional polypeptide into viral structural (gag) and functional (pol) proteins, a process that is essential for the maturation of the infectious HIV particles $[1,2]$. Repression of HIV-1 PR activity could prevent the production of mature and infectious HIV particles, blocking further HIV infection. HIV protease is a centrally symmetric homodimer containing two identical 99 amino acid monomers; the active residues Asp25 and Asp25' are located at the interface between the two monomers [3-5]. The binding on an inhibitor to PR can lead to the inactivation of the enzyme and prevent the infection of the host cell. Thus, the dimeric HIV-1 protease is one of the most attractive targets in the development of antiviral therapeutics. Therefore, in order to design efficient inhibitors, it is critically important to 
investigate the mechanism of the interaction between PR and protease inhibitor (PI) in detail.

Currently, nine antiviral agents that can inhibit HIV-1 protease have been approved by the US Food and Drug Administration (FDA)-including saquinavir, ritonavir, lopinavir, atazanavir, indinavir, amprenavir, and tipranavir-with several others under clinical trial [6]. Due to the short-lived therapeutic benefits of these drugs and the rapid evolution of drug-resistant variants, there is an urgent need to develop antiretroviral drugs with minimal side effects and broad-spectrum activities for current and future wild-type and mutant strains of HIV protease [7, 8]. TMC-126 is an effective nonpeptide inhibitor of HIV-1 protease that is extremely potent against a wide spectrum of HIV protease variants (Fig. 1). Its structure is largely based on that of darunavir (TMC-114) [9], which contains a bistetrahydrofuranyl (bis-THF) urethane and an isostere of sulfonamide [10].

Since it is inconvenient to measure the binding affinities of different PR and inhibitors experimentally, molecular dynamics (MD) simulation can play an important role in investigations of the structural and functional characteristics of biological systems. Using MD simulations, kinetic and thermodynamic data on the simulated system can be obtained. The binding free energy - a very important thermodynamic quantity — can be used to evaluate the stability of a complex. Hence, accurately calculating the binding free energy is crucial when exploring the interactions between proteins and ligands [11]. In order to rapidly evaluate binding free energies, several semiempirical methods such as the molecular mechanics Poisson-Boltzmann surface area (MM-PBSA) and the molecular mechanics generalized Born surface area (MMGBSA) methods have been developed [12]. The MMPBSA approach [13-16], which is based on the MD simulation of the protein-ligand complex of interest in explicit solvent, has been successfully used to describe the protein-ligand binding free energy in rational drug design [17-23]. In this method, the binding free energy is decomposed into the molecular mechanical free energy, the solvation free energy, and entropic contributions. The polar contribution to the solvation energy is evaluated using

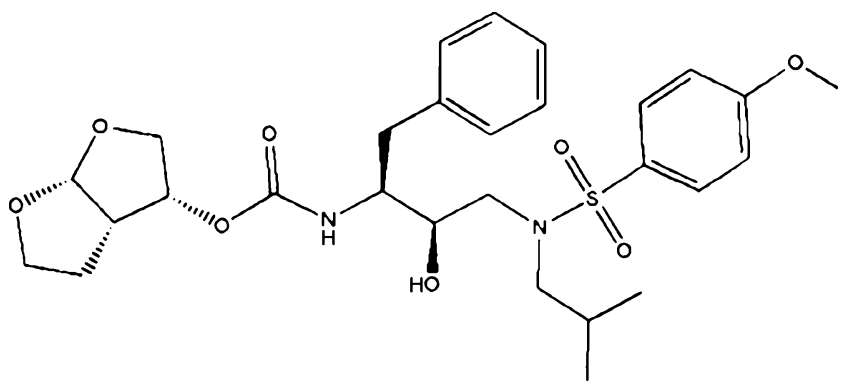

Fig. 1 Structure of TMC-126 the Poisson-Boltzmann (PB) model, or calculated by the generalized Born (GB) model using the MM-GBSA method. The free-energy decomposition involved in the MM-GBSA method can elucidate the contribution of each protease residue to the overall protein-ligand binding free energy at the atomic level [24, 25]. Both MM-PBSA and MM-GBSA have been used to investigate the mechanisms of interaction between different protease inhibitors and different variants of the HIV-1 protease [17]. Previous studies of HIV-1 protease and inhibitors have mostly utilized the MM-PBSA method to obtain the binding free energy, whereas free-energy decomposition methods based on the GB model were selected to analyze the interaction mechanisms $[17,26]$. The GB model is an attractive alternative to the PB model since it is significantly faster and can decompose the binding free energies on a perresidue basis. However, a comparison analysis of the PB and GB models was implemented on the same system that we study in this article. The results showed that the freeenergy changes upon alanine mutation determined by the PB model are smaller and more accurate than those obtained by the GB model [27, 28]. Moreover, when the free-energy decomposition method was performed to investigate the per-residue contributions to the HIV-1 protease by the PB method, the dimerization between the two monomers was barely taken into account.

An extension of the MM-PBSA/MM-GBSA approach based on both the GB and the PB models, computational alanine scanning (CAS) can estimate the free-energy consequences of PR mutations located at the active site, the flap region, and the binding interface from a single MD trajectory. Furthermore, the CAS method allows pairs of residues in the two monomers of PR to be mutated to alanine. Thus, it is a powerful tool for discovering hotspot residues [28-32].

In this work, the relative binding free energies in the complex of PR and TM-126 were obtained using 3 ns MD simulations. The per-residue interactions of the HIV-1 protease and TMC-126 were analyzed by the binding free energy decomposition methods mentioned above. Then the CAS method was implemented to discern 15 important residues of the HIV-1 protease. The results obtained by the CAS method based on the GB model and the PB model were compared and discussed. A full comparison of the decomposition method with the CAS method was also performed in this study.

\section{Theoretical methods}

Initial structure of the complex

The crystal structure of the HIV protease bound to the mutant resistant inhibitor UIC-98038 was obtained from the 
Protein Data Bank (PDB; entry: 3I7E) [10]. The starting structures and force field parameters for the inhibitor were obtained as follows. Special attention was given to the protonation states of Asp25 and Asp25' in the active site. In this work, monoprotonation was adopted, and a proton was added to the oxygen atom OD2 of Asp25 [33]. Considering the importance of water in the binding between PR and the inhibitor, water207 was included in the starting structure $[34,35]$. Partial charges and force field parameters for the inhibitor were generated automatically using the Antechamber program in Amber10 [36, 37]. The atomic charges were derived with the AM1-BCC charge method [38]. The general force field (GAFF) [37] and the standard Amber force field (FF03) [39] were used to obtain parameters such as the Lennard-Jones, torsion, bond, and angle terms for small organic molecules and to describe the parameters of the protein, respectively. All missing hydrogens were added using tleap in Amber10 [36]. To neutralize the charge of the system, $\mathrm{Cl}^{-}$counterions were placed in the grid regions with the largest positive Coulombic potentials around the protease, and then the whole system was soaked in an octahedral periodic box of TIP3P [40] waters. All solute atoms were $10 \AA$ from the edge of the water box.

\section{Molecular dynamics simulations}

All molecular simulations presented in this work were carried out using the Amber10 simulation package and the force field parameters of Cornell et al. [41]. Periodic boundary conditions and a $10 \AA$ cutoff for nonbonded van der Waals (VDW) interactions were applied in our simulations. The particle mesh Ewald (PME) [41] method was employed to account for the long-range electrostatic interactions under periodic boundary conditions. The SHAKE procedure [42] was applied to all atoms covalently bonded to a hydrogen atom. A time step of 2 fs was used to integrate the equations of motion.

In order to remove steric overlap, which produces bad effects between the complex and solvent, two stages of energy minimization were performed: 500 cycles of steepest descent and 2500 cycles of conjugate gradient minimization. First, the water molecules were minimized by keeping the solute fixed with a harmonic constraint of strength $100 \mathrm{kcal} \mathrm{mol}^{-1} \AA^{-2}$. Second, the entire system was minimized without restriction. Subsequently, before the actual MD simulations, the temperature of the system was gradually raised from $0 \mathrm{~K}$ to $300 \mathrm{~K}$ over $100 \mathrm{ps}$, followed by $100 \mathrm{ps}$ of equilibration at $300 \mathrm{~K}$. The initial velocities of atoms were assigned based on a Maxwellian distribution at the starting temperature. Finally, a 3 ns MD simulation was performed at a constant pressure of $1 \mathrm{~atm}$ and constant temperature, and controlled by Langevin dynamics with a collision frequency of $1.0 \mathrm{ps}^{-1}$. The resulting trajectories were analyzed using the ptraj module of Amber10. One snapshot was saved every 5 ps; 200 snapshots were collected from the previous 1000 ps of simulations for post-processing analysis.

\section{MM-PBSA/MM-GBSA approach}

The binding free energy between PR and PI was calculated by the MM-PBSA method according to the following equation:

$\Delta G_{\text {bind }}=G_{\text {complex }}-\left(G_{\text {receptor }}+G_{\text {ligand }}\right)$,

where $G_{\text {complex }}, G_{\text {receptor, }}$ and $G_{\text {ligand }}$ represent the free energies of the complex, receptor, and ligand averaged over snapshots taken from MD trajectories. The free energy of each reactant was estimated as the sum of the molecular mechanical free energy, the solvation free energy, and the contributions from the vibrational, rotational, and translational entropies:

$G=E_{\mathrm{MM}}+G_{\text {solvation }}-T S$.

The molecular mechanical energy $E_{\mathrm{MM}}$ in Eq. 2 was determined with the Sander program from Amber10 software suite according to molecular mechanics with an empirical force field. The topology files thus obtained were further divided into the internal energy of the molecule $\left(E_{\text {int }}\right)$, the electrostatic interactions $\left(E_{\text {ele }}\right)$, and the van der Waals interactions $\left(E_{\mathrm{vdW}}\right)$ :

$E_{\mathrm{MM}}=E_{\text {int }}+E_{\text {ele }}+E_{\mathrm{vdW}}$

$E_{\text {int }}=E_{\text {bond }}+E_{\text {angle }}-E_{\text {torsion }}$.

The internal energy $E_{\text {int }}$ has three contributions: $E_{\text {bond }}$, $E_{\text {angle }}$ and $E_{\text {torsion, }}$, which represent the strain energies in bonds, angles, and torsion angles caused by deviations from their equilibrium values. The electrostatic and van der Waals energies were calculated using the Sander module. The solvation free energy contribution includes polar and nonpolar contributions:

$\Delta G_{\text {sol }}=\Delta G_{\text {polar }}+\Delta G_{\text {nonpolar }}$.

With the PB model, the polar portion $\left(\Delta G_{\text {polar }}\right.$ in Eq. 5) was estimated by the pbsa program of Ambertools under the MM-PBSA approach.

In MM-PBSA calculations, the grid spacing was set to $0.5 \AA$, and the radii of the atoms were taken from the PARSE parameter set [42]. The values of the interior dielectric constant and the exterior dielectric constant were set to 1.0 and 80.0 , respectively. The nonpolar contribution to the solvation free energy, $\Delta G_{\text {nonpolar }}$ in Eq. 5, was calculated from the solvent-accessible surface area (SASA) 
using the LCPO method [43] implemented within Sander, with a probe radius of $1.4 \AA$, according to the equation

$\Delta G_{\text {nonpolar }}=\gamma S A+\beta$,

where the surface tension $\gamma$ and the offset $\beta$ were set to the default values of $0.00542 \mathrm{kcal} /\left(\mathrm{mol} \AA^{2}\right)$ and $0.92 \mathrm{kcal} \mathrm{mol}^{-1}$, respectively.

Unlike the MM-PBSA method, in the MM-GBSA calculation, the polar contribution to the solvation free energy $\left(\Delta G_{\text {polar }}\right.$ in Eq. 5) was calculated with the generalized Born (GB) model implemented in Sander, and the nonpolar contribution ( $\Delta G_{\text {nonpolar }}$ in Eq. 5) was determined with the LCPO method based on the solvent-accessible surface area, as described in Eq. 6, in which the surface tension $\gamma$ and the offset $\beta$ were set to the default values of $0.0072 \mathrm{kcal} /$ $\left(\mathrm{mol} \AA^{2}\right.$ ) and $0.00 \mathrm{kcal} \mathrm{mol}^{-1}$, respectively. Similar to MMPBSA, the values of the interior dielectric constant and the exterior dielectric constant were set to 1.0 and 80.0, respectively [44].

For the calculations of $E_{\mathrm{MM}}, \Delta G_{\mathrm{ELE}}$, and $\Delta G_{\text {nonpolar }}, 200$ snapshots from $2 \mathrm{~ns}$ to $3 \mathrm{~ns}$ were extracted from a single trajectory of the complex at time intervals of about $5 \mathrm{ps}$. In this study, we assumed that the entropy contributions were similar for different HIV protease variants and the ligand. When we calculated the relative binding free energies between them, the entropy contribution was neglected [29].

\section{Binding free-energy decomposition (BFED)}

Due to the time-consuming nature and the high computational demands of the PB calculation, the interactions between the inhibitor and each residue of HIV-1 protease were calculated with a decomposition process based GB model using the mm_pbsa program in Amber10. The per-residue contribution was further decomposed into two parts: one from side chains and the other from the backbone. The binding interactions of each inhibitor-residue pair $\left(\Delta G_{\text {inhibitor-residue }}\right)$ were evaluated using the following equation:

$$
\begin{aligned}
\Delta G_{\text {inhibitor-residue }}= & \Delta E_{\mathrm{vdW}}+\Delta E_{\text {ele }}+\Delta G_{\text {polar }} \\
& +\Delta G_{\text {nonpolar }}
\end{aligned}
$$

We did not take $\Delta E_{\text {int }}$ into account in Eq. 7 for per-atom decomposition because $E_{\text {int }}$ is zero in a single trajectory and the entropy terms are neglected.

The van der Waals contribution $\left(\Delta E_{\mathrm{vdW}}\right)$ and the electrostatic contribution $\left(\Delta E_{\text {ele }}\right)$ in Eq. 7 were computed by the Sander module in Amber10 [36]. In Eq. 7, $\Delta G_{\text {polar }}$ represents the polar interactions between the inhibitor and each protease residue during solvation, and was calculated using the GB model, with the charges taken from the Amber parameter set. The nonpolar solvation contribution ( $\Delta G_{\text {nonpolar }}$ in Eq. 7$)$ was obtained based on the corresponding SASA, as described in Eq. 6 [24].

By summing the atomic energy terms in Eq. 7 over each atom of a given residue, we obtained the contribution of this residue to the total binding free energy. The same snapshots were used to calculate all energy components as well as the total binding free energy.

\section{Computational alanine scanning approach}

The relative binding free-energy changes $\Delta \Delta G_{\text {bind }}$ of different HIV-1 protease variants and the inhibitor were calculated by CAS with the mm_pbsa.pl module in Amber10. $\Delta \Delta G_{\text {bind }}$ was estimated by comparing $\Delta G_{\text {bind }}$ of the alanine mutant to $\Delta G_{\text {bind }}$ of the wild type according to the following equation [29]:

$\Delta \Delta G_{\text {bind }}=\Delta G_{\text {wildtype }}-\Delta G_{\text {mutant }}$.

The key residues of HIV protease were chosen from the binding interface based upon the smallest ligand interaction distances. Since alanine scanning is not suitable for very small (such as glycine) or large residues (the backbone conformations of which differ significantly from that of alanine), prolines and glycines were not selected. The starting atomic coordinates in the alanine mutant structure were obtained by altering the coordinates of the last $1 \mathrm{~ns}$ trajectory. In this calculation, we assumed that the contribution to the change in the entropy of the mutant was not significant, so $\Delta G_{\text {bind }}$ derives from the changes in $\Delta E_{\mathrm{MM}}$ and $\Delta G_{\text {solvate. In }}$ computational alanine scanning, the entropy term can be removed because the entropies of the wild type and its mutants are similar for the same ligand and for similar receptors [29]. Here, the same set of snapshots obtained with the wild-type complex was used to calculate $\Delta G_{\text {bind }}$ for the mutants. The $\Delta \Delta G_{\text {bind }}$ values of the different HIV-1 protease variants and the inhibitor were obtained with the same snapshots of binding free-energy decomposition, according to Eq. 8.

\section{Results and discussion}

Stability and flexibility of the complex

For the complex of HIV-1 protease with inhibitor (TMC126), MD simulations with the particle mesh Ewald (PME) method were performed in explicit water for $3 \mathrm{~ns}$. In order to assess the dynamic stability of the protease complex, Fig. 2 shows the calculated root-mean-square displacement (RMSD) of the backbone atoms from the starting structure of the complex. 


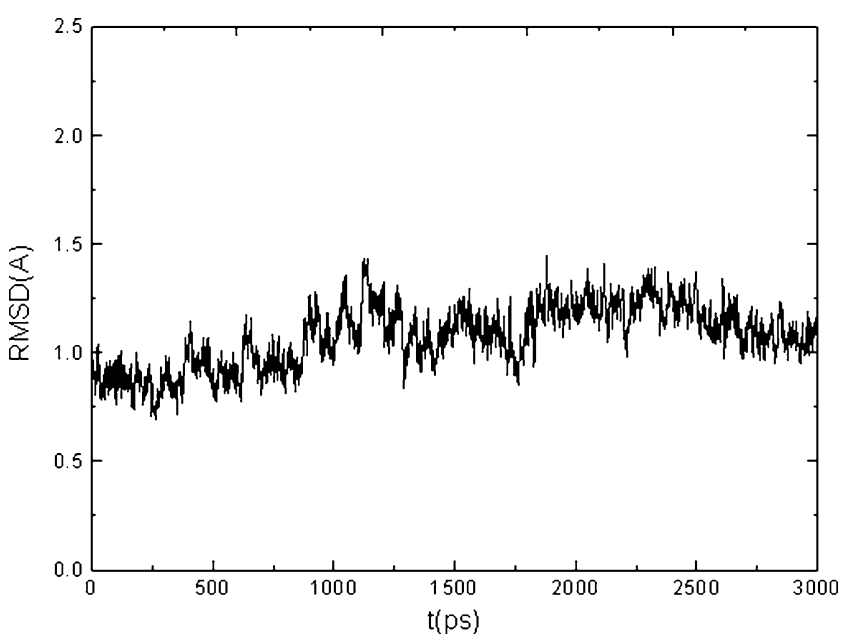

Fig. 2 The root-mean-square deviation (RMSD) of the backbone $\mathrm{C} \alpha$ atoms during the MD simulations of the complex with respect to the initial minimized structure of TMC-126

It is clear from Fig. 2 that a sharp rise is observed during the first 1200 ps. After that, the RMSD values fluctuate between $0.7 \AA$ and $1.5 \AA$. After about $1.8 \mathrm{~ns}$, the RMSD stabilizes and converges to a lower value of $1.1 \AA$, which indicates that the conformation of the complex has reached its equilibrium.

The initial structure and the superimposition of the average structure from the last $1 \mathrm{~ns}$ of snapshots of the complex are shown in Fig. 3. Our results showed that the average backbone RMSD value during the last $1 \mathrm{~ns}$ of MD trajectories was $0.92 \AA$, which indicates that the simulated structure is in good agreement with the experimental results. The last $1 \mathrm{~ns}$ of snapshots were used to calculate the binding free energy, free-energy decomposition, and computational alanine scanning, as described in the following subsections.

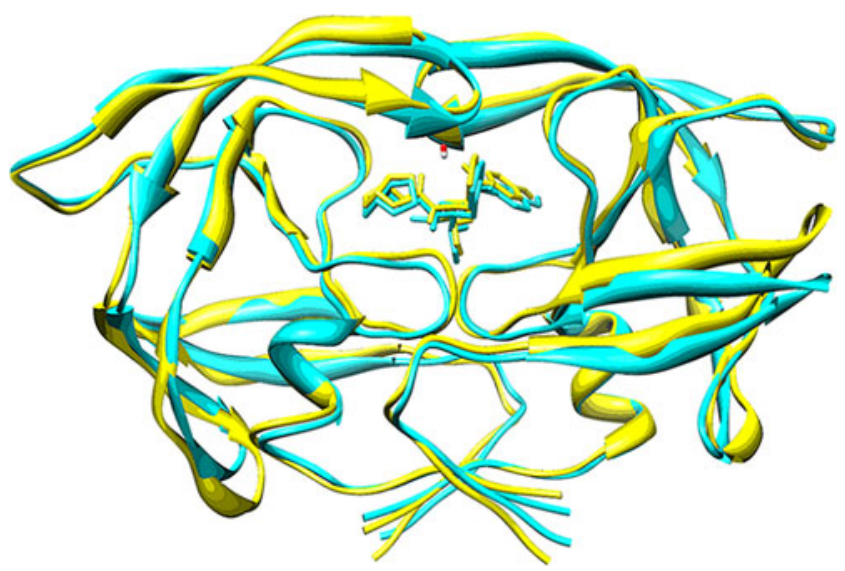

Fig. 3 The average structure from the last $1 \mathrm{~ns}$ of the MD trajectory of the complex of HIV-1 protease with TMC-126 superimposed on the initial structure via the protease's backbone atoms. The initial structure of the complex is shown in cyan, whereas the MD structure of the complex is shown in yellow. The figure was created using Chimera
A detailed analysis of the root-mean-square fluctuation (RMSF) versus the residue number in the complex is illustrated in Fig. 4. As seen from Fig. 4, the regions around Asp25 and Asp25' show analogous RMSF behavior, with a minimum value of $0.3 \AA$. These results are in good agreement with experimental measurements [45], as well as other theoretical reports [46]. In addition to the N- and C-terminal residues, the regions around $17\left(17^{\prime}\right), 41\left(41^{\prime}\right), 52$ $\left(53^{\prime}\right), 67\left(67^{\prime}\right)$, and $81\left(81^{\prime}\right)$ show the biggest dynamic fluctuations. Residues 1-37 and 59-99 in each monomer are defined as the core region, while residues $38-58$ comprise the flap region. The flexibility of the flap region is crucial to the activity of the protease. As seen from Fig. 4, the flap region, especially the flap elbow region (residues 37-42), shows significant flexibility, which was also observed by Zhu et al. [47]. As described in "Theoretical methods," the crystallographic water that bridges the drug TMC-126 and Ile50/Ile50' was included in the initial model. Our results showed that this bridging water was maintained throughout the whole MD simulation.

\section{Binding free energy}

In order to obtain the relative binding free energy and the VDW, electrostatic and solvation energy terms, the MMPBSA and MM-GBSA methods were implemented using a single-trajectory protocol. As described in "Theoretical methods," when we calculated the relative binding free energy between HIV-1 and TMC-126, the entropy contribution was assumed to cancel out completely [48]. The values of the different energy terms shown in Eqs. 1-6 were obtained by averaging 200 snapshots taken from the last $1 \mathrm{~ns}$ of the MD simulation at $5 \mathrm{ps}$ intervals.

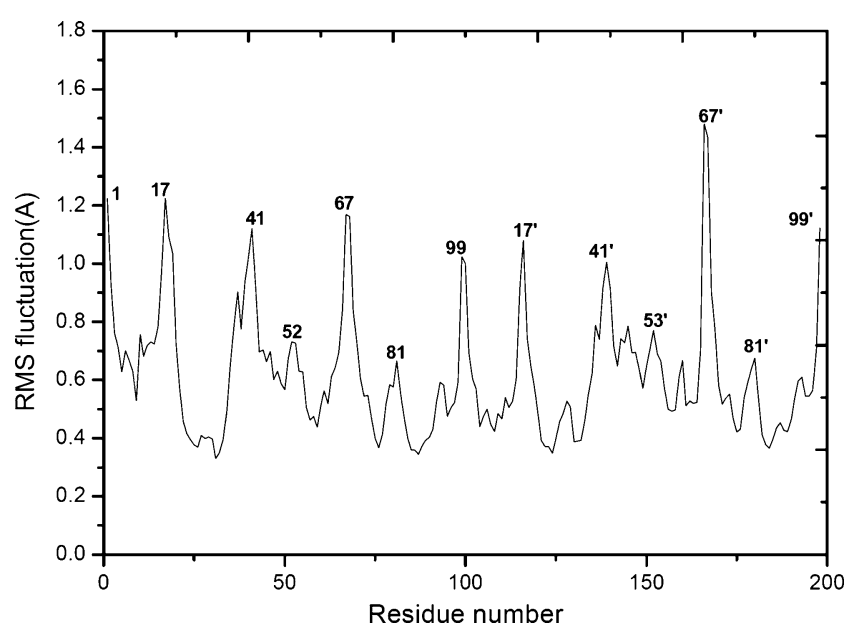

Fig. 4 The root-mean-square fluctuations of the backbone atoms versus the residue number of the HIV-1 protease and TMC-126 complex 
Using Eqs. 1-6, the contributions of the energy components to the relative binding free energies of the receptor, ligand, and complex were calculated, and they are listed in Table 1. Using Eq. 7, with the MM-PBSA and MM-GBSA approaches, the calculated relative binding free energies of TMC-126 with HIV-1 are -37.01 and $-62.37 \mathrm{kcal} \mathrm{mol}^{-1}$, respectively (note that the entropy contribution was assumed to cancel out) [48]. Based on the contributions of the different energy components shown in Table 1, it is clear that the electrostatic interaction and the VDW interaction in the gas phase provide the driving forces for affinity binding. The nonpolar solvation energy provides a slightly favorable contribution to the binding of the inhibitor to PR, whereas the polar solvation energy provides an unfavorable energy component.

\section{Free-energy decomposition}

Analyzing the binding free-energy decomposition and the hydrogen bonds should lead to detailed insights into the binding mechanism for the PR-inhibitor complex. As described in "Theoretical methods," when the MM-GBSA approach is used, the binding free energy is decomposed into per-atom contributions that can be summed over atom groups to obtain the different energy contributions from residues, backbones and side chains $[24,25]$.

Table 2 reports the decomposition of $\Delta G_{\text {bind }}$ on a perresidue basis into the contributions from VDW, electrostatic interactions, polar solvation energy, and nonpolar solvation energy. From Table 2, it is apparent that the calculated protein-inhibitor binding energy $\left(\Delta G_{\text {bind }}\right)$ is greater than $0.12 \mathrm{kcal} \mathrm{mol}^{-1}$, which is extremely helpful in elucidating the binding mechanism of TMC-126 to PR at the atomic level. The calculated binding energies between the asym- metric inhibitor TMC-126 and monomers $\mathrm{A}$ and $\mathrm{B}$ are $18.75 \mathrm{kcal} \mathrm{mol}^{-1}$ and $14.49 \mathrm{kcal} \mathrm{mol}^{-1}$, respectively, which are very close to the values calculated by Zhang et al. [49]. The contributions of the flap region (38-58) and the active site region (residue 23-32) are $10.4 \mathrm{kcal} \mathrm{mol}^{-1}$ and $12.72 \mathrm{kcal} \mathrm{mol}^{-1}$, respectively, which correspond to $31.39 \%$ and $38.39 \%$ of the contribution of PR to the binding; the flap elbow region makes a contribution of $0.02 \mathrm{kcal} \mathrm{mol}^{-1}$, corresponding to $0.1 \%$ of the total binding.

Table 2 shows that the residues Ala28, Ile50, Ile84', Gly27, Ile50', Ala28', Ile47', and Gly49 contribute more than $1.5 \mathrm{kcal} \mathrm{mol}^{-1}$ to the binding. These residues are mostly from the flap region (residues 38-58) and the active site region (residues 23-32). As we know, the flap region and the active site region are important regions for the binding [47]. Ala28 contributes $-4.65 \mathrm{kcal} \mathrm{mol}^{-1}$ to the binding affinity, most of which derives from its backbone $\left(-3.96 \mathrm{kcal} \mathrm{mol}^{-1}\right)$. Ala28' also makes a rather significant contribution of $-1.77 \mathrm{kcal} \mathrm{mol}^{-1}$, and its backbone contributes $-1.11 \mathrm{kcal} \mathrm{mol}^{-1}$. Ile50 and Ile50' also provide large contributions to the binding: $-2.63 \mathrm{kcal} \mathrm{mol}^{-1}$ and $-2.08 \mathrm{kcal} \mathrm{mol}^{-1}$, respectively. Unlike Ala28/Ala28', instead of their backbones contributing most, their side chains (with VDW interactions) are the main contributors to the binding. Their nonpolar solvation energies (1.03 and $0.71 \mathrm{kcal} \mathrm{mol}^{-1}$ ) appear to be unfavorable for binding. Similar to Ile50/Ile 50', the side-chain contribution dominates for the other two isoleucine residues, Ile84 and Ile27. As shown in Table 2, Ile47 has a greater contribution from its backbone than from its side chain, and its binding occurs mainly through the VDW interaction. More than a half of the contribution of Gly49' $\left(-1.61 \mathrm{kcal} \mathrm{mol}^{-1}\right)$ originates from the side chain $\left(-0.91 \mathrm{kcal} \mathrm{mol}^{-1}\right)$, according to the calculated free-energy decomposition.
Table 1 The binding free energy components of the protein-inhibitor complex (HIV1-TCM126), as calculated using MMPBSA methods (unit: $\mathrm{kcal} \mathrm{mol}^{-1}$ )

${ }^{\text {a }}$ Components: $E_{\text {ele }}$ Coulombic energy; $E_{\mathrm{vdW}}$ VDW energy;

$E_{\mathrm{MM}}=E_{\mathrm{ele}}+E_{\mathrm{vdW}} ; G_{\text {polar,PB }}$ polar solvation energy; $G_{\text {nonpolar,PB }}$ nonpolar solvation energy; $G_{\text {sol- }}$ vation, $\mathrm{PB}=G$ polar, $\mathrm{PB}+G_{\text {nonpolar, } \mathrm{PB}}$; $G_{\text {subtotal, } \mathrm{PB}}=E_{\mathrm{MM}}+G_{\text {solvation, } \mathrm{PB}}$

${ }^{\mathrm{b}}$ Average of 200 snapshots

c Standard error of the mean value

\begin{tabular}{|c|c|c|c|c|c|c|c|c|}
\hline \multirow[t]{2}{*}{ Component $^{\mathrm{a}}$} & \multicolumn{2}{|l|}{ Complex } & \multicolumn{2}{|l|}{ PR } & \multicolumn{2}{|c|}{ TMC-126 } & \multicolumn{2}{|l|}{ Delta } \\
\hline & Mean $^{\mathrm{b}}$ & $\mathrm{Std}^{\mathrm{c}}$ & Mean & Std & Mean & Std & Mean & Std \\
\hline$E_{\text {ele }}$ & -4103.6 & 54.13 & -3939.2 & 53.79 & -131.6 & 2.19 & -32.8 & 5.65 \\
\hline$E_{\mathrm{vdW}}$ & -833.22 & 20.58 & -771.16 & 19.85 & 5.72 & 2.64 & -67.77 & 3.59 \\
\hline$E_{\mathrm{MM}}$ & -539.47 & 64.38 & -441.34 & 63.28 & 2.44 & 6.14 & -100.57 & 5.6 \\
\hline$G_{\text {nonpolar,PB }}$ & 56.83 & 0.77 & 58.83 & 0.78 & 5.29 & 0.05 & -7.29 & 0.09 \\
\hline$G_{\text {polar }, \mathrm{PB}}$ & -2380.55 & 45.03 & -2411.06 & 45.05 & -40.33 & 1.29 & 70.84 & 4.17 \\
\hline$G_{\text {solvation,PB }}$ & -2323.72 & 44.59 & -2352.23 & 44.62 & -35.04 & 1.28 & 63.55 & 4.15 \\
\hline$G_{\text {subtotal, } \mathrm{PB}}$ & -2863.19 & 41.54 & -2793.57 & 40.5 & -32.61 & 5.97 & -37.01 & 4.68 \\
\hline$G_{\text {nonpolar,GB }}$ & 74.27 & 1.03 & 76.93 & 1.04 & 5.8 & 0.07 & -8.46 & 0.12 \\
\hline$G_{\text {polar,GB }}$ & -2398.71 & 47.09 & -2409.76 & 47.04 & -35.61 & 1.58 & 46.66 & 4.42 \\
\hline$G_{\text {solvation,GB }}$ & -2324.44 & 46.5 & -2332.83 & 46.45 & -29.81 & 1.56 & 38.2 & 4.4 \\
\hline$G_{\text {subtotal,GB }}$ & -2863.91 & 39.26 & -2774.17 & 38.56 & -27.37 & 6.11 & -62.37 & 4.46 \\
\hline
\end{tabular}


Table 2 Decomposition of $\Delta G_{\text {bind }}$ on a per-residue basis into contributions from the van der Waals energy $\left(\Delta E_{\mathrm{vdW}}\right)$, electrostatic interaction energy $\left(\Delta E_{\text {ele }}\right)$, nonpolar solvation free energy $\left(\Delta G_{\mathrm{polar}, \mathrm{GB}}\right)$ and polar free energy $\left(\Delta G_{\text {nonpolar }}\right)$ (units: kcal mol $\left.{ }^{-1}\right)$
$\mathrm{S}, \mathrm{B}$, and $\mathrm{T}$ represent the sidechain residue, monomer $\mathrm{B}$, and total (monomers $\mathrm{A}$ and $\mathrm{B}$ ) contributions, respectively

\begin{tabular}{|c|c|c|c|c|c|c|c|}
\hline Residue & $\Delta E_{\mathrm{vdW}}$ & $\Delta E_{\text {ele }}$ & $\Delta G_{\text {polar,GB }}$ & $\Delta G_{\text {nonpolar }}$ & $\mathrm{S} \Delta G_{\text {subtotal }}$ & B $\Delta G_{\text {subtotal }}$ & $\mathrm{T} \Delta G_{\text {subtotal }}$ \\
\hline Ala28 & -2.55 & -2.01 & 0.09 & -0.18 & -0.69 & -3.96 & -4.65 \\
\hline Ile50 & -2.3 & -1.18 & 1.03 & -0.18 & -2.16 & -0.47 & -2.63 \\
\hline Ile84' & -1.85 & -0.2 & 0.01 & -0.17 & -2.13 & -0.09 & -2.22 \\
\hline Gly27 & -1.37 & 0.0 & -0.66 & -0.1 & -0.71 & -1.43 & -2.14 \\
\hline Ile $50^{\prime}$ & -1.72 & -0.9 & 0.71 & -0.17 & -1.83 & -0.26 & -2.08 \\
\hline Ala28' & -1.46 & 0.42 & -0.55 & -0.18 & -0.66 & -1.11 & -1.77 \\
\hline Ile47' & -1.53 & 0.31 & -0.3 & -0.16 & -1.56 & -0.12 & -1.69 \\
\hline Gly49' & -0.81 & -1.67 & 0.96 & -0.08 & -0.91 & -0.7 & -1.61 \\
\hline Ile84 & -1.15 & 0.22 & -0.33 & -0.13 & -1.27 & -0.11 & -1.39 \\
\hline Gly49 & -1.05 & -1.29 & 1.09 & -0.12 & -0.75 & -0.63 & -1.38 \\
\hline Val82' & -0.97 & 0.06 & -0.34 & -0.09 & -1.2 & -0.15 & -1.35 \\
\hline Val32' & -0.78 & 0.0 & -0.43 & -0.07 & -1.15 & -0.11 & -1.27 \\
\hline Gly27' & -0.61 & 0.35 & -0.67 & -0.04 & -0.41 & -0.57 & -0.98 \\
\hline Leu23' & -0.7 & -0.14 & -0.04 & -0.04 & -0.79 & -0.13 & -0.92 \\
\hline Arg87 & -0.19 & -2.19 & 1.58 & 0.0 & -0.74 & -0.07 & -0.8 \\
\hline Ile47 & -0.89 & 0.36 & -0.14 & -0.11 & -0.74 & -0.05 & -0.79 \\
\hline Arg8' & -0.87 & -1.24 & 1.48 & -0.16 & -0.73 & -0.06 & -0.79 \\
\hline Pro81' & -0.63 & -0.17 & 0.11 & -0.09 & -0.61 & -0.16 & -0.78 \\
\hline Asp25 & -1.1 & 1.44 & -0.95 & -0.07 & -0.68 & 0.0 & -0.68 \\
\hline Val32 & -0.49 & -0.03 & -0.13 & -0.03 & -0.64 & -0.04 & -0.68 \\
\hline Val82 & -0.38 & -0.08 & -0.05 & -0.05 & -0.52 & -0.05 & -0.57 \\
\hline Leu23 & -0.38 & 0.12 & -0.24 & -0.04 & -0.44 & -0.09 & -0.54 \\
\hline Asp29 & -1.6 & 1.5 & -0.3 & -0.11 & 0.78 & -1.3 & -0.52 \\
\hline Pro81 & -0.35 & -0.1 & 0.04 & -0.04 & -0.4 & -0.05 & -0.46 \\
\hline Leu76' & -0.39 & 0.1 & -0.11 & -0.02 & -0.4 & -0.02 & -0.42 \\
\hline Thr26 & -0.2 & 0.3 & -0.48 & 0.0 & 0.13 & -0.52 & -0.38 \\
\hline Asn83' & -0.09 & 0.05 & -0.3 & 0.0 & -0.03 & -0.32 & -0.35 \\
\hline Gly86 & -0.09 & -0.35 & 0.13 & 0.0 & -0.23 & -0.07 & -0.31 \\
\hline Gly86' & -0.08 & 0.27 & -0.4 & 0.0 & -0.22 & 0.01 & -0.21 \\
\hline Leu24' & -0.09 & 0.15 & -0.27 & 0.0 & -0.01 & -0.19 & -0.2 \\
\hline Val56' & -0.06 & 0.02 & -0.13 & 0.0 & -0.19 & 0.01 & -0.17 \\
\hline Leu76 & -0.14 & -0.03 & 0.01 & 0.0 & -0.15 & -0.01 & -0.16 \\
\hline Asn83 & -0.05 & -0.03 & -0.08 & 0.0 & -0.02 & -0.14 & -0.16 \\
\hline Arg87' & -0.07 & 1.82 & -1.9 & 0.0 & -0.08 & -0.07 & -0.15 \\
\hline Asp30 & -1.27 & 1.99 & -0.75 & -0.08 & 0.23 & -0.35 & -0.12 \\
\hline
\end{tabular}

\section{Computational alanine scanning}

The computational alanine scanning (CAS) method was implemented to acquire $\Delta \Delta G_{\text {bind }}$ by mutating residues of proteins to alanine. HIV-1 is a homodimer protease consisting of two identical monomers. In terms of the binding decomposition method, the CAS method can mutate the same residue in each monomer simultaneously, so that we can gain insight into the function of the residue in the homodimer.

In order to perform CAS, we first need to select which residue pairs can be mutated to alanine. Our criteria for selecting these mutated pairs were that the chosen residues should make significant contributions to the binding, or that they should be located in a crucial area such as the binding interface, pocket, or flap region. As mentioned in "Theoretical methods," the CAS method does not work well for very small or very big residue mutations. The mutation of proline [50] to alanine sometimes leads to significant conformational changes [29]. In this study, based on our selection criteria, and by combining the residue locations and the results reported in Table 3, 15 pairs of residues were chosen for mutation to alanine in the CAS method: Arg8, Leu23, Leu24, Thr26, Asp29, Asp30, Val32, Ile47, Ile50, Val56, Leu76, Val82, Asn83, Ile84, and Arg87. In order to make this method practical, we assumed that local changes do not 
Table 3 The computational alanine scanning results for the HIV-1 protease and TMC-126 complex

\begin{tabular}{|c|c|c|c|c|c|c|c|c|c|c|}
\hline Contribution & Arg8Ala & Std & Leu23Ala & Std & Leu24Ala & Std & Thr26Ala & Std & Asp29Ala & Std \\
\hline$\Delta \Delta E_{\mathrm{ele}}$ & 0.85 & 1.65 & 0.03 & 0.03 & -0.03 & 0.01 & -0.08 & 0.09 & 3.26 & 2.48 \\
\hline$\Delta \Delta E_{\mathrm{vdW}}$ & -1.83 & 0.35 & -1.73 & 0.43 & -0.03 & 0.00 & -0.07 & 0.01 & -1.39 & 0.29 \\
\hline$\Delta \Delta E_{\mathrm{MM}}$ & -0.98 & 1.68 & -1.7 & 0.42 & -0.06 & 0.01 & -0.15 & 0.09 & 1.87 & 2.53 \\
\hline$\Delta \Delta G_{\text {nonpolar,PB }}$ & -0.13 & 0.04 & 0.12 & 0.03 & 0.0 & 0.01 & 0.0 & 0.01 & -0.02 & 0.05 \\
\hline$\Delta \Delta G_{\text {polar,PB }}$ & 0.77 & 1.68 & -0.12 & 0.79 & 0.01 & 0.28 & -0.07 & 0.31 & -2.06 & 2.31 \\
\hline$\Delta \Delta G_{\text {solvation,PB }}$ & 0.64 & 1.67 & 0.0 & 0.77 & 0.01 & 0.28 & -0.07 & 0.31 & -2.08 & 2.31 \\
\hline$\Delta \Delta G_{\text {subtotal,PB }}$ & -0.33 & 1.02 & -1.69 & 0.85 & -0.04 & 0.28 & -0.21 & 0.28 & -0.19 & 1.54 \\
\hline$\Delta \Delta G_{\text {nonpolar,GB }}$ & -0.17 & 0.05 & 0.16 & 0.04 & 0.0 & 0.01 & 0.0 & 0.01 & -0.02 & 0.07 \\
\hline$\Delta \Delta G_{\text {polar,GB }}$ & -1.33 & 1.41 & -1.02 & 0.14 & -0.05 & 0.04 & 0.78 & 0.24 & 0.25 & 2.24 \\
\hline$\Delta \Delta G_{\text {solvation, GB }}$ & -1.5 & 1.42 & -0.86 & 0.14 & -0.05 & 0.04 & 0.78 & 0.24 & 0.25 & 2.24 \\
\hline$\Delta \Delta G_{\text {solvation, GB }}$ & -1.5 & 1.42 & -0.86 & 0.14 & -0.05 & 0.04 & 0.78 & 0.24 & 0.23 & 2.24 \\
\hline$\Delta \Delta G_{\text {subtotal,GB }}$ & -2.48 & 0.71 & -2.57 & 0.46 & -0.12 & 0.04 & 0.63 & 0.19 & 2.1 & 1.06 \\
\hline Contribution & Asp30Ala & std & Val32Ala & std & Ile47Ala & std & Ile50Ala & std & Val56Ala & Std \\
\hline$\Delta \Delta E_{\mathrm{ele}}$ & -1.11 & 2.37 & 0.01 & 0.24 & 0.25 & 0.20 & -0.09 & 6.94 & 0.09 & 0.06 \\
\hline$\Delta \Delta E_{\mathrm{vdW}}$ & -0.86 & 0.31 & -1.4 & 0.46 & -2.85 & 0.50 & -3.84 & 4.51 & -0.12 & 0.02 \\
\hline$\Delta \Delta E_{\mathrm{MM}}$ & -1.97 & 2.34 & -1.39 & 0.49 & -2.6 & 0.56 & -3.93 & 6.42 & -0.03 & 0.06 \\
\hline$\Delta \Delta G_{\text {nonpolar,PB }}$ & -0.01 & 0.03 & 0.09 & 0.02 & 0.09 & 0.03 & 0.15 & 0.12 & 0.0 & 0.01 \\
\hline$\Delta \Delta G_{\text {polar,PB }}$ & 4.35 & 2.08 & -1.53 & 0.71 & 0.85 & 0.59 & 2.17 & 5.07 & 0.03 & 0.19 \\
\hline$\Delta \Delta G_{\text {solvation,PB }}$ & 4.34 & 2.08 & -1.44 & 0.71 & 0.94 & 0.58 & 2.32 & 5.07 & 0.03 & 0.19 \\
\hline$\Delta \Delta G_{\text {subtotal,PB }}$ & 2.38 & 1.93 & -2.82 & 0.80 & -1.65 & 0.78 & -1.6 & 5.54 & 0.01 & 0.19 \\
\hline$\Delta \Delta G_{\text {nonpolar,GB }}$ & -0.01 & 0.03 & 0.12 & 0.02 & 0.12 & 0.04 & 0.2 & 0.16 & 0.0 & 0.01 \\
\hline$\Delta \Delta G_{\text {polar,GB }}$ & 3.59 & 2.00 & -1.32 & 0.33 & -0.24 & 0.17 & -0.89 & 5.33 & -0.58 & 0.11 \\
\hline$\Delta \Delta G_{\text {solvation, GB }}$ & 3.58 & 2.01 & -1.2 & 0.34 & -0.12 & 0.18 & -0.69 & 5.34 & -0.58 & 0.11 \\
\hline$\Delta \Delta G_{\text {subtotal,GB }}$ & 1.61 & 0.86 & -2.59 & 0.49 & -2.72 & 0.53 & -4.62 & 5.57 & -0.61 & 0.08 \\
\hline Contribution & Leu76Ala & std & Val82Ala & std & Asn83Ala & std & Ile84Ala & std & Arg87Ala & std \\
\hline$\Delta \Delta E_{\mathrm{ele}}$ & 0.01 & 0.04 & -0.38 & 0.25 & 0.12 & 0.11 & 0.26 & 0.28 & -0.64 & 1.08 \\
\hline$\Delta \Delta E_{\mathrm{vdW}}$ & -0.85 & 0.32 & -1.61 & 0.41 & -0.03 & 0.00 & -4.6 & 0.82 & -0.36 & 0.04 \\
\hline$\Delta \Delta E_{\mathrm{MM}}$ & -0.84 & 0.30 & -1.99 & 0.43 & 0.09 & 0.11 & -4.34 & 0.88 & -1.0 & 1.09 \\
\hline$\Delta \Delta G_{\text {nonpolar,PB }}$ & 0.04 & 0.03 & 0.05 & 0.02 & 0.0 & 0.01 & 0.15 & 0.05 & 0.0 & 0.01 \\
\hline$\Delta \Delta G_{\text {polar,PB }}$ & 0.8 & 0.90 & 0.35 & 0.35 & -0.15 & 0.16 & 1.73 & 1.13 & -0.77 & 1.07 \\
\hline$\Delta \Delta G_{\text {solvation,PB }}$ & 0.83 & 0.90 & 0.4 & 0.34 & -0.16 & 0.16 & 1.88 & 1.10 & -0.77 & 1.07 \\
\hline$\Delta \Delta G_{\text {subtotal,PB }}$ & 0.01 & 0.90 & -1.57 & 0.53 & -0.06 & 0.12 & -2.45 & 1.49 & -1.76 & 0.62 \\
\hline$\Delta \Delta G_{\text {nonpolar,GB }}$ & 0.05 & 0.04 & 0.07 & 0.03 & 0.0 & 0.01 & 0.2 & 0.07 & 0.0 & 0.01 \\
\hline$\Delta \Delta G_{\text {polar,GB }}$ & -0.13 & 0.09 & -0.9 & 0.31 & -0.68 & 0.13 & -1.15 & 0.31 & -0.63 & 0.98 \\
\hline$\Delta \Delta G_{\text {solvation, GB }}$ & -0.08 & 0.11 & -0.83 & 0.31 & -0.69 & 0.13 & -0.95 & 0.30 & -0.64 & 0.98 \\
\hline$\Delta \Delta G_{\text {subtotal,GB }}$ & -0.92 & 0.27 & -2.82 & 0.50 & -0.6 & 0.10 & -5.29 & 0.91 & -1.63 & 0.40 \\
\hline
\end{tabular}

Std standard error (units: kcal $\mathrm{mol}^{-1}$ )

impact on the global conformation of the PR and the total binding modes for the binding of PR to inhibitor. Such an assumption has been proven to be applicable for most mutations according to various alanine scanning mutagenesis experiments [51].

The results obtained with the CAS approach for 15 pairs of residues of PR are shown in Table 3. The changes in the energy terms (VDW interactions, electrostatic interactions, the polar and nonpolar solvation free energies) upon alanine mutation are also listed in Table 3. According to Eq. 8, negative values of $\Delta \Delta G_{\text {bind }}$ indicate unfavorable substitutions. In contrast, positive $\Delta \Delta G_{\text {bind }}$ values suggest that the alanine residue at the mutated position is more favorable for binding.

As can be seen from Table 3, the binding free energies drop considerably when the six critical residues (Leu23, Val32, Ile47, Ile50, Val82, and Ile84) are mutated to alanine. On the contrary, the $\Delta \Delta G_{\text {subtotal,GB }}$ values of Val26, Asp29, and Asp30 are all positive, which indicates that the binding between protease and TMC-126 is influenced when these 
residues are mutated to alanine. These results are in good agreement with those obtained from the free-energy decomposition method.

\section{Interactions between HIV-1 protease and inhibitor}

In this section, we analyze some PR residues that are key to the binding to the inhibitor, based on the results obtained using BFED and CAS and the hydrogen bond data listed in Table 4.

The interactions between PR and the inhibitor based on the average structure from the MD simulations are plotted in Fig. 5. In order to investigate the hydrogen bonds during MD simulations, the results of a dynamic analysis of hydrogen bonds based on the trajectories of the MD simulations are listed in Table 4. Some of the hydrogen bonds are also indicated in Fig. 5.

The BEFD-calculated results show that the favorable residues mainly come from six groups around Ala28/ Ala28', Ile50/Ile50', and Ile84/Ile84'. Table 4 shows that the residues that make significant contributions to the binding are all hydrophobic amino acids. According to the results shown in Table 2, the major force that drives the inhibitor to most of the residues in PR is VDW interactions, especially for the essential residues. This conclusion is consistent with the binding free energies shown in Table 1.

As shown in Table 2, Ala28 contributes most of the binding affinity. The main driving forces for the binding of the inhibitor to Ala28 are the VDW energy $\left(-2.55 \mathrm{kcal} \mathrm{mol}^{-1}\right)$ and electrostatic energy $\left(-2.01 \mathrm{kcal} \mathrm{mol}^{-1}\right)$, which originate in the C-H... $\pi$ interactions between the bis-tetrahydrofuran (THF) and the alkyl of Ala28, and in the C-H...O interactions of the side-chain atoms of Ala28 with the oxygen atoms of the bisTHF and inhibitor, respectively. The interaction between

Table 4 Hydrogen bonds for the last 1000 ps of the trajectory

\begin{tabular}{|c|c|c|c|}
\hline \multicolumn{2}{|c|}{ Hydrogen bonds } & \multirow[t]{2}{*}{$\%$ Occupied } & \multirow[t]{2}{*}{ Distance } \\
\hline Donor & Receptor & & \\
\hline Asp25-OD1 & Ala28-N-H & 98.80 & $2.851(0.10)$ \\
\hline Asp25'-OD1 & PI-O22-H & 98.60 & $2.670(0.12)$ \\
\hline $\mathrm{PI}-\mathrm{O} 4$ & Asp29'-N-H & 95.2 & $3.004(0.17)$ \\
\hline $\mathrm{PI}-\mathrm{O} 2$ & Asp30'-N-H & 61.2 & $3.203(0.17)$ \\
\hline $\mathrm{PI}-\mathrm{O} 2$ & Asp29'-N-H & 43.2 & $3.220(0.17)$ \\
\hline \multicolumn{4}{|c|}{ Hydrogen bonds between WAT and $\mathrm{PR}^{\mathrm{b}}$ and $\mathrm{PI}^{\mathrm{a}}$} \\
\hline WAT-O & Ile 50-N-H & 91.2 & $3.084(0.18)$ \\
\hline WAT-O & Ile $50^{\prime}-\mathrm{N}-\mathrm{H}$ & 90.2 & $3.022(0.17)$ \\
\hline PI-O11 & WAT-O-H1 & 86.60 & $2.787(0.14)$ \\
\hline $\mathrm{PI}-\mathrm{O} 26$ & WAT-O-H2 & 83.20 & $2.748(0.15)$ \\
\hline
\end{tabular}

${ }^{\text {a }}$ HIV-1 protease

${ }^{\mathrm{b}}$ HIV-1 protease inhibitor
Ala28' and the inhibitor is similar to that of Ala28, in that the main driving force for the binding of Ala28' to the inhibitor is the van der Waals energy $\left(-1.46 \mathrm{kcal} \mathrm{mol}^{-1}\right)$ from the $\mathrm{C}-\mathrm{H} . . . \pi$ interactions between the phenoxymethyl of the inhibitor and the alkyl of Ala28'. In this case, the favorable polar solvation energy $\left(-0.55 \mathrm{kcal} \mathrm{mol}^{-1}\right)$ is mostly countered by the unfavorable electrostatic energy $\left(0.42 \mathrm{kcal} \mathrm{mol}^{-1}\right)$.

Although Asp25 and Asp25' are located at the active site as catalytic aspartic acids, monoprotonated Asp25 contributes only $-0.68 \mathrm{kcal} \mathrm{mol}^{-1}$ with an unfavorable electrostatic interaction $\left(1.44 \mathrm{kcal} \mathrm{mol}^{-1}\right)$ to the total binding affinity, and Asp25' makes an unfavorable contribution $\left(2.02 \mathrm{kcal} \mathrm{mol}^{-1}\right)$ to the binding affinity due to the combination of a strongly unfavorable polar solvation energy $\left(11.48 \mathrm{kcal} \mathrm{mol}^{-1}\right)$ and a highly favorable electrostatic energy $\left(-9.51 \mathrm{kcal} \mathrm{mol}^{-1}\right)$. As shown in Table 4, the OD1 of Asp25 and Asp25' form hydrogen bonds with Ala28 and the inhibitor with occurrence rates of $>98 \%$, which suggests that the Ala28 pair make a favorable contribution to the binding that stabilizes the complex.

Although the PR is symmetrical, it is clear from Table 4 that the calculated contributions of Asp29 and Asp29' are different: negative $\left(-0.52 \mathrm{kcal} \mathrm{mol}^{-1}\right)$ and positive $\left(0.14 \mathrm{kcal} \mathrm{mol}^{-1}\right)$, respectively. Similar behavior is also found for the residues Asp30 (-0.12 kcal mol $\left.{ }^{-1}\right)$ and Asp30' $\left(0.36 \mathrm{kcal} \mathrm{mol}^{-1}\right)$. The results in Table 4 show that Asp29 and Asp30 form stable hydrogen bonds with the oxygen of the bis-THF in the inhibitor. The donor-acceptor distances for these pairs and their corresponding occupancies are $3.004 \AA$ and $3.204 \AA, 95.2 \%$ and $61.2 \%$, respectively. On the other hand, Asp29' and Asp30' do not form hydrogen bonds with the inhibitor. Therefore, the hydrogen bonds make favorable contributions to the VDW interactions of Asp29 and Asp30 with the inhibitor, which suggests that the bis-THF group in TMC-126 plays an important role in the binding with the PR. In addition, as shown in Table 3 , the calculated $\Delta \Delta G_{\text {subtotal,GB }}$ values of the residue pairs Asp29Ala and Asp30Ala are 2.1 and $1.61 \mathrm{kcal} \mathrm{mol}^{-1}$ respectively, which means that the side chains of Asp29 and Asp30 make unfavorable contributions to the binding affinity. The calculated $\Delta \Delta E_{\text {ele }}$ of Asp29Ala and the $\Delta \Delta G_{\text {polar,GB }}$ of Asp30Ala are $3.26 \mathrm{kcal} \mathrm{mol}^{-1}$ and $3.59 \mathrm{kcal} \mathrm{mol}^{-1}$, respectively, indicating that the electrostatic interactions of their side chains have a significant effect on the binding to the inhibitor.

The calculated results listed in Table 3 show that there is a significant loss of binding free energy when the four critical residue pairs Ile47/Ile47' Ile50/Ile50', Ile84/Ile84', and Val82/Val82' are mutated to alanine. The CAScalculated results show that the residues Ile50/Ile 50 ' have a significant effect on the binding, a $-4.71 \mathrm{kcal} \mathrm{mol}^{-1}$ reduction in $\Delta \Delta G_{\text {subtotal,GB}}$, which is in fair agreement with the side-chain contribution $\left(-3.99 \mathrm{kcal} \mathrm{mol}^{-1}\right)$ obtained 
Fig. 5 Geometries of ten residues of HIV-1 protease that participate in some of the strongest interactions with TMC-126, based on the average structure from the last $1 \mathrm{~ns}$ of MD simulation

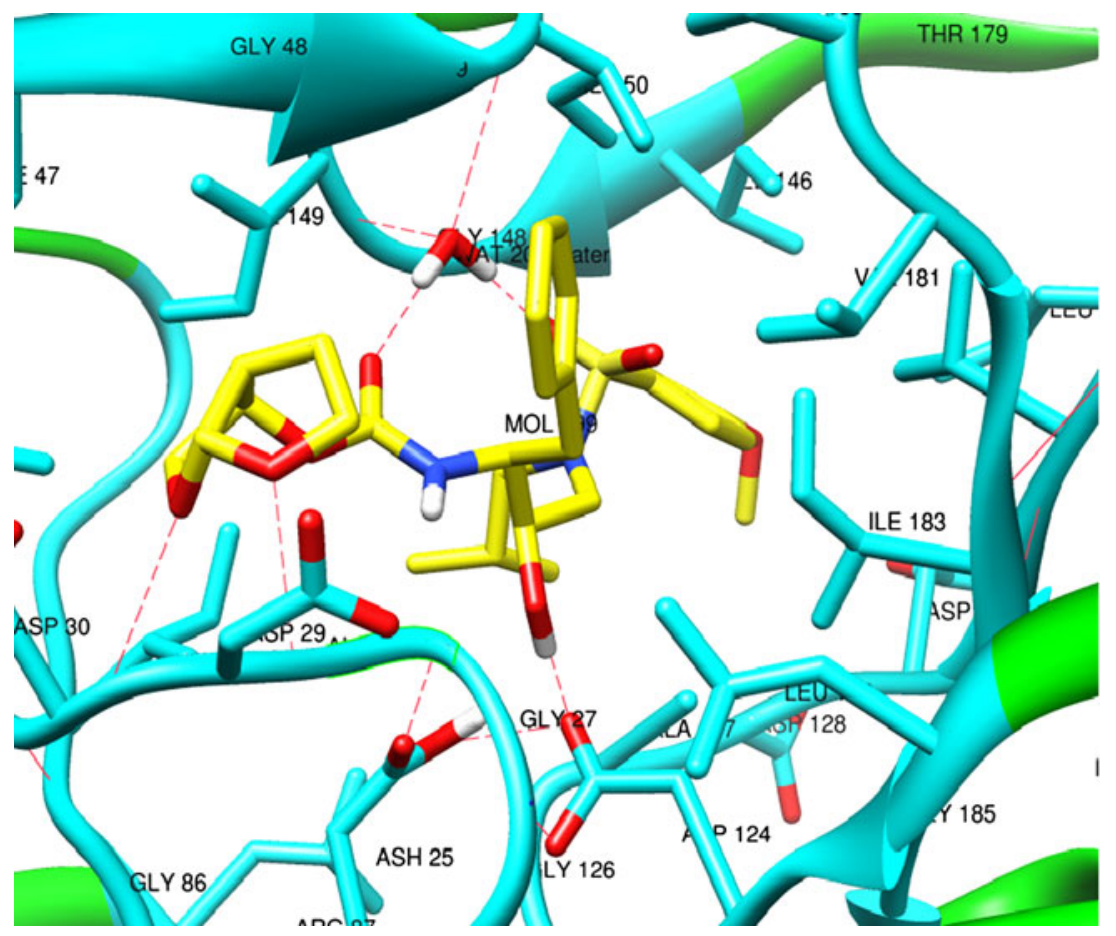

using the BFED method (Tables 2 and 3). As shown in Table 3, the favorable contributions of Ile 50 and Ile $50^{\prime}$ to the binding energy originate mainly from the VDW interaction $\left(-3.84 \mathrm{kcal} \mathrm{mol}^{-1}\right)$ in Table 3 . A possible reason for this is that the side-chain alkyls of Ile50 and Ile 50' form some $\mathrm{C}-\mathrm{H} \ldots \mathrm{H}-\mathrm{C}$ interactions with the inhibitor. In addition, the hydrogen atoms connect with the backbone nitrogen atoms of Ile50 and Ile50', forming strong hydrogen bonds with the oxygen of the WAT207 (Table 4).

As can be seen from Tables 2 and 3, the interactions of Ile84/Ile84' and Ile47' with the inhibitor are similar to that of Ile50/Ile 50'. Moreover, the contact of the alkyls of Ile47' and Ile84' with the phenyl group of the inhibitor, which results in $\mathrm{C}-\mathrm{H} . . . \pi$ interactions, can also add strong van der Waals interactions to the binding. These results indicate that the VDW interactions significantly favor the binding of these isoleucine residues.

It is well known that the crystal water molecule WAT207 plays an important role in PR-inhibitor binding, since it can generally form four hydrogen bonds with Ile50/Ile50' and TMC-126 [26]. As shown in Table 4, the occupancy rates of these four hydrogen bonds are higher than $80 \%$, which suggest that these hydrogen bonds are extraordinarily stable during MD simulations, and that the WAT207 act as a conduit to connect the inhibitor with PR.

\section{Comparisons between the CAS and the BFED methods}

The calculated results obtained by computational alanine scanning using the MM-PBSA and MM-GBSA approaches are shown in Table 5. The regression between the calculated $\Delta \Delta G_{\text {subtotal.PB }}$ and $\Delta \Delta G_{\text {subtotal.GB }}$ values for the 15 pairs of residues is depicted in Fig. 6. From Fig. 6, the correlation coefficient between the calculated $\Delta \Delta G_{\text {subtotal.PB }}$ and $\Delta \Delta G_{\text {subtotal.GB }}$ values was found to be 0.76 for 15 mutations. As shown in Table 5, the values of $\Delta \Delta G_{\text {bind }}$ obtained by the PB model are clearly smaller than those yielded by the GB model, except for Val32Ala and Arg87Ala. Since the standard deviations obtained by the GB model are a little bit smaller than those given by the PB model, it appears that in this system, the PB model is more sensitive to the atomic coordinates than the GB model when calculating contributions to the solvation energy. These conclusions are consistent with the results reported by $\mathrm{Li}$ et al. [52].

In order to compare the computational alanine scanning (CAS) approach with the binding free-energy decomposition (BFED) approach, in Table 5 we sum the calculated $\Delta \Delta G_{\text {subtotal }}$ values of the same 15 pairs of residues corresponding to chains A and B using the BFED method, and the calculated $\Delta \Delta G_{\text {subtotal,GB }}$ results for the 15 pairs of residues obtained by the CAS method.

The per-residue contributions calculated using the BFED method include the contributions from the backbone and the side chain. As far as the CAS method is concerned, the backbone does not generally change, because only $\mathrm{C} \gamma$ is replaced by a methyl in the mutated topology file. Consequently, $\Delta \Delta G_{\text {subtotal }}$ mainly reflects the contribution from the side chain of the residue. Thus, the side-chain contributions from the residues in monomers 
Table 5 Summation of $\Delta G_{\text {subtotal }}$ obtained by free-energy decomposition for the same residues in chain A and chain B of the complex, and the $\Delta \Delta G_{\text {subtotal,GB }}$ values obtained by computational alanine scanning for the 15 pairs of residues (units: $\mathrm{kcal} \mathrm{mol}^{-1}$ )

\begin{tabular}{|c|c|c|c|c|c|c|c|}
\hline Residue & $\mathrm{A}^{\mathrm{a}} \mathrm{S}^{\mathrm{c}} \Delta G_{\text {subtotal }}$ & A $\Delta G_{\text {subtotal }}$ & B ${ }^{\mathrm{b}} \mathrm{S} \Delta G_{\text {subtotal }}$ & B $\Delta G_{\text {subtotal }}$ & $\mathrm{T}^{\mathrm{d}} \mathrm{S} \Delta G_{\text {subtotal }}$ & T $\Delta G_{\text {subtotal }}$ & $\Delta \Delta G_{\text {subtotal,GB }}$ \\
\hline Arg8 & 0.01 & -0.06 & -0.73 & -0.79 & -0.72 & -0.85 & -2.48 \\
\hline Leu23 & -0.44 & -0.54 & -0.79 & -0.92 & -1.23 & -1.46 & -2.57 \\
\hline Leu24 & -0.01 & -0.11 & -0.01 & -0.2 & -0.02 & -0.31 & -0.12 \\
\hline Thr26 & 0.13 & -0.38 & 0.11 & -0.09 & 0.24 & -0.47 & 0.63 \\
\hline Asp29 & 0.78 & -0.52 & 0.13 & 0.14 & 0.91 & -0.38 & 2.1 \\
\hline Asp30 & 0.23 & -0.12 & 0.29 & 0.36 & 0.52 & 0.24 & 1.61 \\
\hline Val32 & -0.64 & -0.68 & -1.15 & -1.27 & -1.79 & -1.95 & -2.59 \\
\hline Ile47 & -0.74 & -0.79 & -1.56 & -1.69 & -2.3 & -2.48 & -2.72 \\
\hline Ile 50 & -2.16 & -2.63 & -1.83 & -2.08 & -3.99 & -4.71 & -4.62 \\
\hline Val56 & -0.08 & -0.07 & -0.19 & -0.17 & -0.27 & -0.24 & -0.61 \\
\hline Leu76 & -0.15 & -0.16 & -0.4 & -0.42 & -0.55 & -0.58 & -0.92 \\
\hline Val82 & -0.52 & -0.57 & -1.2 & -1.35 & -1.72 & -1.92 & -2.82 \\
\hline Asn83 & -0.02 & -0.16 & -0.03 & -0.35 & -0.05 & -0.51 & -0.6 \\
\hline Ile84 & -1.27 & -1.39 & -2.13 & -2.22 & -3.4 & -3.61 & -5.29 \\
\hline $\operatorname{Arg} 87$ & -0.74 & -0.8 & -0.08 & -0.13 & -0.82 & -0.93 & -1.64 \\
\hline
\end{tabular}

a A represents $\Delta G_{\text {subtotal }}$ for the residues in chain $\mathrm{A}$

b $\mathrm{B}$ represents $\Delta G_{\text {subtotal }}$ for the residues in chain B

c $\mathrm{S}$ represents $\Delta G_{\text {subtotal }}$ for the side chains

${ }^{\mathrm{d}} \mathrm{T}$ represents the summation $\Delta \Delta G_{\text {subtotal }}$ for the residues in chains $\mathrm{A}$ and $\mathrm{B}$

$\mathrm{A}$ and $\mathrm{B}$ obtained using the BFED method and the $\Delta \Delta G_{\text {subtotal,GB }}$ results obtained by the CAS method are compared and shown in Fig. 7. The correlation coefficient between $\Delta \Delta G_{\text {subtotal }}$ for the side chain calculated by the BFED method and $\Delta \Delta G_{\text {subtotal,GB }}$ calculated by the CAS method is 0.94 for the 15 mutants of residues, which indicates that the CAS method allows useful insight into the contribution of the side chain. Zoete and Michielin studied different systems using both BFED and CAS, and concluded that both of these methods can achieve consistent results [32], which is in good agreement with our calculated results.

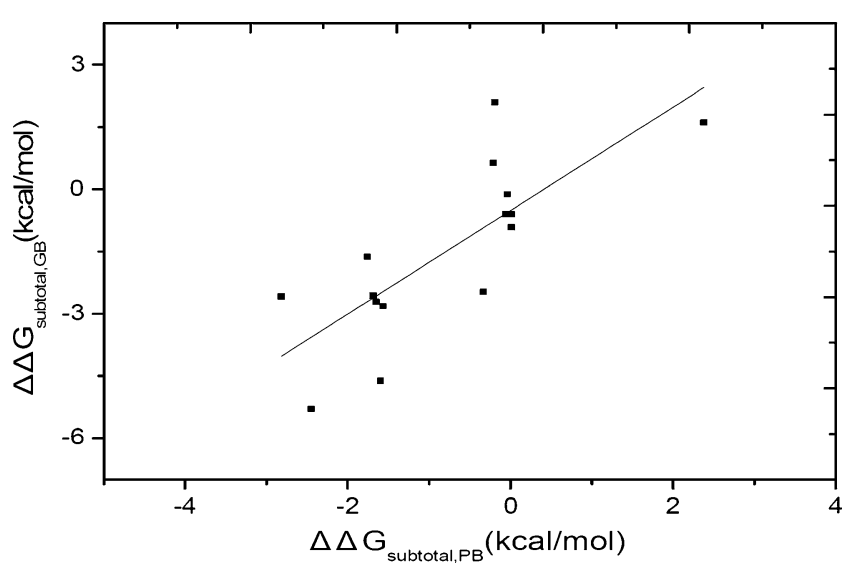

Fig. 6 Regression between the calculated $\Delta \Delta G_{\text {subtotal,PB }}$ and $\Delta \Delta G_{\text {subtotal,PB }}$ values obtained by computational alanine scanning for the 15 residues
Our results showed that the correlation between BFED and CAS is better than that between GB and PB, as calculated by the CAS method. A possible reason for this is that both BEED and CAS are based upon the GB model in our study.

Both the BFED and the CAS methods have been widely used to identify the hotspots of receptor-ligand complexes reliably and to obtain further insight into their binding and related functional information [32]. Using the analysis of the BFED and CAS methods, it can be concluded that

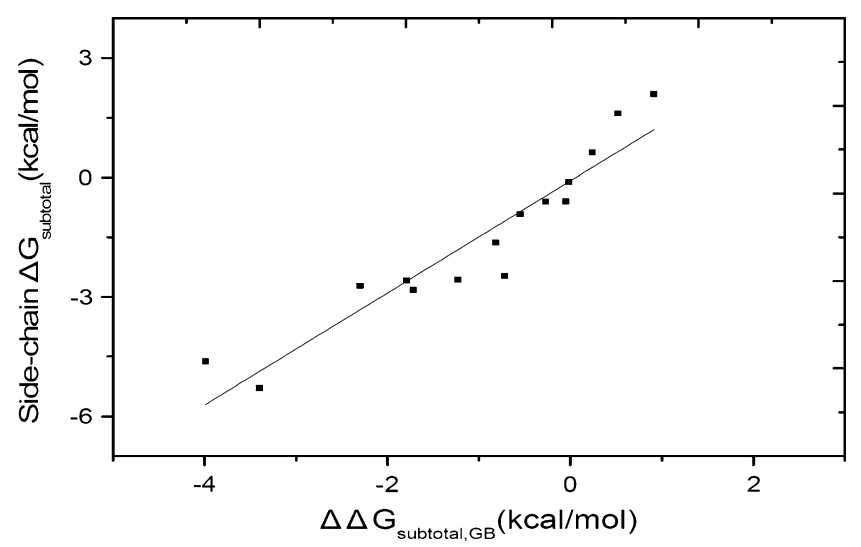

Fig. 7 Regression between the calculated $\Delta \Delta G_{\text {subtotal,GB }}$ obtained by computational alanine scanning and the $\Delta G_{\text {subtotal }}$ for the side chain, obtained by free-energy decomposition for the 15 pairs of residues of HIV-1 protease 
although these two methods are different, they are complementary to some extent.

First, the BFED method is based upon the GB model; as far as the CAS method is concerned, both PB and GB models are applied to estimate the binding free-energy differences. Consequently, owing to the distinctness of the models' principles, the results calculated based on the PB model of CAS methods are more accurate than those calculated with the BFED methods. Compared to BFED, the CAS calculations are time-consuming. Second, by applying the BFED approach at an atomistic level, the per-atom contributions can be summed over atomic groups such as residues, backbones, and side chains, in order to obtain their contributions to the binding free energy of the receptor-ligand complex. Simultaneously, the BFED approach allows us to decompose each residue's binding free energy conveniently into different energetic components, such as VDW interactions, electrostatic interactions, and nonpolar solvation energy. On the other hand, the CAS approach requires separate, time-consuming calculations to perform a detailed study of protein-protein interactions at the residue level, and focuses on the impact of side chains on the binding affinity, since it is only employed to evaluate the side-chain contributions of the residues of intest that are mutated into alanine in the topology files. Unfortunately, the CAS method can only be applied to specific residues: not very small residues (such as glynine) or residues that would induce significant global conformational changes (such as proline and cysteine). Third, it is worth mentioning that the CAS approach provides a chance to investigate the influence of several mutants in the complex on the binding affinity, because it allows more than one residue to be mutated to alanine when the topology files are prepared. In contrast to the BFED method, the CAS method provides a preferable insight into systems in which several mutations exist simultaneously; in particular, it can be applied to the dimer and even more complicated systems, such as the HIV-1 protease homodimer and inhibitor complex. As the CAS method substitutes alanine for other residues, this approach can also be regarded as an effective tool to analyze the drug resistance caused by mutagenesis, which could result in improvements to drug design and better guidance for new experimental investigations. In addition, the results obtained by CAS can be compared directly to the experimental data on mutagenesis. However, the CAS approach is not suitable for detecting the binding mechanisms of complexes in which the binding affinity originates from the backbones.

\section{Conclusions}

The binding free energy of the complex of HIV-1 protease and TMC-126 was calculated using the MM-PBSA and MM-GBSA methods based on decomposition of the energy at an atomic level on the basis of the GB model. The computational alanine scanning method based on the GB and PB models was applied to this complex in order to investigate the different contributions of HIV-1 protease residues when binding to TMC-126.

The binding mechanism of PR with TMC-126 was investigated by structural analysis, by calculating the free energy and decomposing the inhibitor-residue interaction, and computational alanine scanning. The favorable interactions and the driving forces in the binding of the inhibitor to PR are the van der Waals and electrostatic forces, which mainly come from six groups around Ala28/Ala28', Ile50/ Ile50', and Ile84/Ile84. Ala28 mainly contributes to the binding affinity. The contributions of Asp29 and Asp30 to the binding in chains $\mathrm{A}$ and $\mathrm{B}$ are significantly different because they form hydrogen bonds with bis-THF of the inhibitor, which suggests that the bis-THF group in TMC-126 plays an important role in the binding of the PR. The VDW energy significantly favors binding for isoleucine residues such as Ile47, Ile50 and Ile84. The flap region and the active site region of the PR are crucial to its binding affinity, contributing about $69.78 \%$ of the total binding affinity. The crystal water molecule acts as a bridging medium between the inhibitor and PR by forming four hydrogen bonds among the residues Ile50/Ile50' and TMC-126.

Based on the correlation coefficients obtained from regression analyses relating to different theoretical methods and models, both the BFED and the CAS methods have particular advantages and weaknesses when investigating the binding mechanism. BFED is a rapid and convenient approach. It does not need to consider the global change and can prove the effects of both the backbone and side chains for each residue. However, the CAS method can provide preferable insight into the resistance of mutagenesis and the binding affinities between residues in dimic and multimeric proteins and inhibitors. To conclude, these two complementary methods provide a useful way to determine the hotspot residues and to investigate the binding affinity incisively. We expect that this work will provide some helpful insights into the future of drug design with potent inhibitors.

Acknowledgments This work is supported by the 973 Fund of the Chinese Ministry of Science and Technology (2010CB934504) and the Natural Science Fund of China.

\section{References}

1. Wlodawer A (2002) Rational approach to AIDS drug design through structural biology. Annu Rev Med 53:595-614

2. Navia MA, Fitzgerald PMD, Mckeever BM, Leu CT, Heimbach JC, Herber WK, Sigal IS, Darke PL, Springer JP (1989) 3Dimensional structure of aspartyl protease from human immunodeficiency virus Hiv-1. Nature 337:615-620 
3. Prabu-Jeyabalan M, Nalivaika E, Schiffer CA (2002) Substrate shape determines specificity of recognition for HIV-1 protease: analysis of crystal structures of six substrate complexes. Structure 10:369-381

4. Swain AL, Miller MM, Green J, Rich DH, Schneider J, Kent SBH, Wlodawer A (1990) X-ray crystallographic structure of a complex between a synthetic protease of human immunodeficiency virus-1 and a substrate-based hydroxyethylamine inhibitor. Proc Natl Acad Sci USA 87:8805-8809

5. Wlodawer A, Miller M, Jaskolski M, Sathyanarayana BK, Baldwin E, Weber IT, Selk LM, Clawson L, Schneider J, Kent SBH (1989) Conserved folding in retroviral proteases-crystal structure of a synthetic HIV-1 protease. Science 245:616-621

6. Barbaro G, Scozzafava A, Mastrolorenzo A, Supuran CT (2005) Highly active antiretroviral therapy: current state of the art, new agents and their pharmacological interactions useful for improving therapeutic outcome. Curr Pharm Des 11:1805-1843

7. Chen RX, Quinones-Mateu ME, Mansky LM (2004) Drug resistance, virus fitness and HIV-1 mutagenesis. Curr Pharm Des 10:4065-4070

8. Clavel F, Hance AJ (2004) Medical progress: HIV drug resistance. New Engl J Med 350:1023-1035

9. Surleraux DLNG, Tahri A, Verschueren WG, Pille GME, Kock HA, Jonckers THM, Peeters A, Meyer DS, Azijn H, Pauwels R, Bethune MP, King NM, Prabu-Jeyabalan M, Schiffer CA, Wigerinck PBTP (2005) Discovery and selection of TMC114, a next generation HIV-1 protease inhibitor. J Med Chem 48:18131822

10. Ghosh AK, Kulkarni S et al (2009) Design, synthesis, proteinligand X-ray structure, and biological evaluation of a series of novel macrocyclic human immunodeficiency virus-1 protease inhibitors to combat drug resistance. J Med Chem 52:7689-7705

11. Stewart AAJ, Andrew M (2006) Molecular dynamics: survey of methods for simulating the activity of proteins. Chem Rev 6:1589-1615

12. Kollman PA (1993) Free-energy calculations - applications to chemical and biochemical phenomena. Chem Rev 7:2395-2417

13. Kollman PA, Massova I, Reyes C, Kuhn B, Huo SH, Chong L, Lee M, Lee T, Duan Y, Wang W, Donini O, Cieplak P, Srinivasan J, Case DA, Cheatham TE (2000) Calculating structures and free energies of complex molecules: combining molecular mechanics and continuum models. Acc Chem Res 33:889-897

14. Lee MR, Duan Y, Kollman PA (2000) Use of MM-PB/SA in estimating the free energies of proteins: application to native, intermediates, and unfolded villin headpiece. Proteins 39:309-316

15. Wang W, Kollman PA (2000) Free energy calculations on dimer stability of the HIV protease using molecular dynamics and a continuum solvent model. J Mol Biol 303:567-582

16. Wang W, Kollman PA (2001) Computational study of protein specificity: the molecular basis of HIV-1 protease drug resistance. Proc Natl Acad Sci USA 98:14937-14942

17. Hou TJ, Yu R (2007) Molecular dynamics and free energy studies on the wild-type and double mutant HIV-1 protease complexed with amprenavir and two amprenavir-related inhibitors: mechanism for binding and drug resistance. J Med Chem 50:1177-1188

18. Swanson JM, Henchman RH, McCammon JA (2004) Revisiting free energy calculations: a theoretical connection to MM/PBSA and direct calculation of the association free energy. Biophys $J$ 86:67-74

19. Xu Y, Wang RX (2006) A computational analysis of the binding affinities of FKBP12 inhibitors using the MM-PB/SA method. Proteins 64:1058-1068

20. Wang J, Morin P, Wang W, Kollman PA (2001) Use of MM-PBSA in reproducing the binding free energies to HIV-1RT of TIBO derivates and predicting the binding mode to HIV-1 RT of
Efavirenz by docking and MM-PBSA. J Am Chem Soc 123:5221-5230

21. Kuhn B, Gerber P, Schulz-Gasch T, Stahl M (2005) Validation and use of the MM-PBSA approach for drug discovery. J Med Chem 48:4040-4048

22. Luo C, Xu L, Zheng S, Luo X, Shen J, Jiang H, Liu X, Zhou M (2005) Computational analysis of molecular basis of 1:1 interactions of NRG-1beta wild-type and variants with ErbB3 and ErbB4. Proteins 59:742-756

23. Huo S, Wang J, Cieplak P, Kollman PA, Kuntz ID (2002) Molecular dynamics and free energy analyses of cathepsin Dinhibitor interactions: insight into structure-based ligand design. J Med Chem 45:1412-1419

24. Gohlke H, Kiel C, Case DA (2003) Insights into protein-protein binding by binding free energy calculation and free energy decomposition for the Ras-Raf and Ras-RalGDS complexes. J Mol Biol 330:891-913

25. Zoete V, Meuwly M, Karplus M (2005) Study of the insulin dimerization: binding free energy calculations and per-residue free energy decomposition. Proteins 61:79-93

26. Chen JZ, Zhang SL, Liu XG, Zhang QG (2010) Insights into drug resistance of mutations $\mathrm{D} 30 \mathrm{~N}$ and $\mathrm{I} 50 \mathrm{~V}$ to HIV-1 protease inhibitor TMC-114: free energy calculation and molecular dynamic simulation. J Mol Model 16:459-468

27. Massova I, Kollman PA (2000) Combined molecular mechanical and continuum solvent approach (MM-PBSA/GBSA) to predict ligand binding. Perspect Drug Disc Des 18:113-135

28. Huo S, Massova I, Kollman PA (2002) Computational alanine scanning of the 1:1 human growth hormone-receptor complex. J Comput Chem 23:15-27

29. Massova I, Kollman PA (1999) Computational alanine scanning to probe protein-protein interactions: a novel approach to evaluate binding free energies. J Am Chem Soc 121:8133-8143

30. Lillian TC, William CS, Jed WP, Vijay SP (2006) Kinetic computational alanine scanning: application to p53 oligomerization. J Mol Biol 357:1039-1049

31. Zoete V, Meuwly M (2006) Importance of individual side chains for the stability of a protein fold: computational alanine scanning of the insulin monomer. J Comput Chem 27:1843-1857

32. Zoete V, Michielin O (2007) Comparison between computational alanine scanning and per-residue binding free energy decomposition for protein-protein association using MM-GBSA: application to the TCR-p-MHC complex. Protein 67:1026-1047

33. Chen XN, Tropsha A (1995) Relative binding free energies of peptide inhibitors of HIV-1 protease: the influence of the active site protonation state. J Med Chem 38:42-48

34. Tie Y, Boross PI, Wang YF, Gaddis L, Hussain AK, Leshchenko S, Ghosh AK, Louis JM, Harrison RW, Weber IT (2004) High resolution crystal structures of HIV-1 protease with a potent nonpeptide inhibitor [UIC-94017] active against multidrug-resistant clinical strains. J Mol Biol 338:341-352

35. Kovalevsky AY, Tie Y, Liu F, Boross PI, Wang YF, Leshchenko S, Ghosh AK, Harrison RW, Weber IT (2006) Effectiveness of nonpeptide clinical inhibitor TMC-114 on HIV-1 protease with highly drug resistant mutations D30N, I50V, and L90M. J Med Chem 49:1379-1387

36. Case DA, Darden TA, Cheatham TE, Simmerling CL, Wang J, Duke RE, Luo R, Crowley M, Walker RC, Zhang W, Merz KM, Wang B, Hayik S, Roitberg A, Seabra G, Kolossváry I, Wong KF, Paesani F, Vanicek J, Wu X, Brozell SR, Steinbrecher T, Gohlke H, Yang L, Tan C, Mongan J, Hornak V, Cui G, Mathews DH, Seetin MG, Sagui C, Babin V, Kollman PA (2008) AMBER 10 University of California, San Francisco

37. Wang JM, Wolf RM, Caldwell JW, Kollman PA, Case DA (2004) Development and testing of a general Amber force field. J Comput Chem 25:1157-1174 
38. Jakalian A, Bush BL, Jack DB, Bayly CI (2000) Fast, efficient generation of high-quality atomic charges. AM1-BCC model: I. Method. J Comput Chem 21:132-146

39. Duan Y, Wu C, Chowdhury S, Lee MC, Xiong GM, Zhang W, Yang R, Cieplak P, Luo R, Lee T, Caldwell J, Wang JM, Kollman PA (2003) Point-charge force field for molecular mechanics simulations of proteins based on condensed-phase quantum mechanical calculations. J Comput Chem 24:19992012

40. Jorgensen WL, Chandrasekhar J, Madura JD, Impey RW, Klein ML (1983) Comparison of simple potential functions for simulating liquid water. J Chem Phys 79:926-935

41. Darden T, York D, Pedersen L (1993) Particle mesh Ewald - an N. $\log -[\mathrm{N}]$ method for Ewald sums in large systems. J Chem Phys 98:10089-10092

42. Ryckaert JP, Ciccotti G, Berendsen HJC (1977) Numerical integration of Cartesian equations of motion of a system with constraintsmolecular dynamics of n-alkanes. J Comput Phys 23:327-341

43. Weiser J, Shenkin PS, Still WC (1999) Approximate atomic surfaces from linear combinations of pairwise overlaps (LCPO). J Comput Chem 20:217-230

44. Onufriev A, Bashford D, Case DA (2000) Modification of the generalized Born model suitable for macromolecules. J Phys Chem B 104:3712-3720

45. Freedberg DI, Wang YX, Stahl SJ, Kaufman JD, Wingfield PT, Kiso Y, Torchia DA (1998) Flexibility and function in HIV protease: dynamics of the HIV-1 protease bound to the asymmetric inhibitor kynostatin 272 [KNI-272]. J Am Chem Soc 120:79167923

46. Zoete V, Michielin O, Karplus M (2002) Relation between sequence and structure of HIV-1 protease inhibitor complexes: a model system for the analysis of protein flexibility. J Mol Biol 315:21-52

47. Zhu ZW, Schuster DI, Tuckerman ME (2003) Molecular dynamics study of the connection between flap closing and binding of fullerene-based inhibitors of the HIV-1 protease. Biochemistry 42:1326-1333

48. Ishima RD, Freedberg I et al (1999) Flap opening and dimerinterface flexibility in the free and inhibitor-bound HIV protease, and their implications for function. Structure 7:1047-1055

49. Zhang DW, Zhang JZH (2005) Full quantum mechanical study of binding of HIV-1 protease drugs. Int J Quantum Chem 103:246257

50. Clackson T, Ultsch MH, Wells JA, de Vos AM (1998) Structural and functional analysis of the 1:1 growth hormone: receptor complex reveals the molecular basis for receptor affinity. $\mathrm{J}$ Mol Biol 277:1111-1128

51. Cunningham BC, Wells JA (1989) High-resolution epitope mapping of hGH-receptor interactions by alanine-scanning mutagenesis. Science 244:1081-1085

52. Li T, Froeyen M, Herdewijn P (2008) Computational alanine scanning and free energy decomposition for E. coli type I signal peptidase with lipopeptide inhibitor complex. J Mol Graph Model $26: 813-823$ 